\title{
Adaptive Self-configurable Rectifier for Extended Operating Range of Piezoelectric Energy Harvesting
}

\author{
Zheng Jun Chew, Member, IEEE, and Meiling Zhu, Member, IEEE
}

\begin{abstract}
This paper presents an adaptive selfconfigurable rectifier to increase the range of vibration excitation that a piezoelectric energy harvesting power management circuit (PMC) can operate with. The proposed circuit configures itself as a voltage doubler (VD) to increase the low voltage of piezoelectric energy harvesters to meet the minimum operating voltage of the PMC. This allows energy harvesting from low voltage range that is not viable using a full-wave bridge (FB) rectifier. When the piezoelectric voltage increases and causes the voltage amplified by the VD to approach the maximum operating voltage of the PMC, the proposed circuit reconfigures itself to $F B$ rectifier that does not amplify the voltage. This allows operation at a higher voltage range that is restricted by the VD topology. Two different reference voltages each for the VD topology and the FB rectifier topology with low-pass filtering are used to ensure correct switching of the rectifier topology. The proposed control circuit can cold start from a piezoelectric voltage of $1.2 \mathrm{~V}$ in the VD topology to amplify the voltage to allow an existing PMC that needs a minimum input voltage higher than $1.2 \mathrm{~V}$ to operate while consuming 240$410 \mathrm{nW}$ of power. With the two-reference-voltages control method, $20 \%$ more energy can be transferred through the PMC than the one without it.
\end{abstract}

Index Terms-adaptive, low power, piezoelectric energy harvesting, rectifier, self-configurable, switching.

\section{INTRODUCTION}

$\mathrm{E}$ NERGY harvesting from energy sources such as thermal [1], solar [2], fluid flow [3], and motion from the ambient environments [4] into electrical energy for low power devices has garnered increasing research attention recently. With the growing interest in using wireless sensors for condition or structural health monitoring [5]-[8], energy harvesting is seen as a promising way to power the ubiquitous wireless sensor nodes without relying solely on batteries that require regular

Manuscript received November 7, 2018; revised January 29, 2019; revised February 22, 2019; accepted March 4, 2019. This work was supported by Innovate UK, through the project Multi-source Power Management to Enable Autonomous Micro Energy Harvesting Systems. All data are provided in full in the results section of this paper.

Z. J. Chew and M. Zhu are with the College of Engineering, Mathematics and Physical Sciences, University of Exeter, Exeter EX4 4QF U.K. (e-mail: z.j.chew@exeter.ac.uk; m.zhu@exeter.ac.uk). replacement, which is usually associated with high costs [9]. One of the most prevalent sources that can be harvested in engineering structures and machinery is vibration [5]-[8].

Piezoelectric energy harvesters (PEHs) are widely used in vibration energy harvesting due to their high energy densities and self-contained power generation capabilities [10]. Due to the nature of vibrations and PEHs, the electrical output from PEHs is generally ac [4], [11]. Since most electronic circuits require dc voltage, the ac output of PEHs needs to be rectified before feeding it to a PMC for maximum power transfer and voltage regulation [12]. Both passive [11], [13] and active [14], [15] FB rectifiers have been widely used to convert the ac output from PEHs to dc. Passive diode based rectifiers are common due to their simplicity and robustness against high voltage [11], [13], [16], although there are some voltage and power losses due to the inherent diode forward voltage drop $V_{\mathrm{F}}$. The diode forward voltage drop also limits the capability of the rectifiers in converting very low ac voltage.

Active rectifiers have been proposed to overcome the issues related to $V_{\mathrm{F}}$ in passive diode based rectifiers when the ac voltage from the energy harvester is low [14], [17]. However, in energy harvesting applications without an external power source, energy harvesters still need to produce a certain level of voltage to power the active rectifiers for them to operate properly [15]. Therefore, the minimum voltage required by active rectifiers could still be similar to $V_{\mathrm{F}}$ in passive rectifiers [15], [17]. The maximum operating voltage of active rectifiers is also limited by the fabrication technologies or components to, for example, 5-10 V [17], [18], which is far lower than the voltage that PEHs can possibly generate [6], [13].

Passive diodes have also been used as VDs to amplify the rectified piezoelectric voltage [19]. This is particularly useful to boost the low voltage amplitude of PEHs due to low vibration excitation or small-size of PEHs [20]. However, VDs can only increase the piezoelectric voltage. Any further increment of the voltage that is already very high when the vibration excitation is high requires the circuit components to have a very high voltage rating to withstand and handle the electrical energy. This can be very expensive or even not feasible for certain fabrication technologies [19], [21].

Methods that have been proposed to extend the operation range of piezoelectric energy harvesting include using two PEHs that can be connected in series [22] or connecting equally-split electrodes of a monolithic PEH in series [23] to 
increase their output voltages. These methods involve the use of multiple PEHs or customized transducers, which might limit their wide application due to constraints such as cost and also their integration with existing PMCs.

This paper herein presents an adaptive self-configurable diode based rectifying circuit to allow piezoelectric energy harvesting over an extended range than a PMC with only a fixed rectifier topology. The proposed rectifier switches between VD and FB rectifier depending on the piezoelectric voltage, adapting to both low and high vibration excitation respectively. To ensure the proposed circuit switches its topologies correctly, reference voltage switching with lowpass filtering is introduced. The proposed circuit was integrated into an existing PMC and tested to demonstrate its operating voltage range, switching of the rectifier topologies, and power consumption.

\section{SYSTEM DESCRIPTION}

\section{A. Concept of the Configurable Rectifier}

The proposed method is to add a switch to a conventional passive diode based FB rectifier to make the rectifying circuit configurable between two different topologies of FB rectifier and VD. By adding a switch in parallel with diode $D_{4}$ of the FB rectifier shown in Fig. 1, the rectifier can be configured as a FB rectifier or a VD. If the switch is opened, the rectifying circuit remains as the FB rectifier since the open switch is not a conduction path and does not change the overall topology of the FB rectifier. When the switch is closed, $D_{4}$ will be bypassed. The terminal of the PEH that is connected to the anode of $D_{3}$ and cathode of $D_{4}$ will be shorted to the anode of both $D_{1}$ and $D_{4}$, which is usually a ground reference. Given that there is usually a smoothing capacitor $C_{\mathrm{i}}$ at the output of the rectifier [11], [13]-[15], which tends to hold some charges, a voltage will present at the cathode of $D_{2}$ and $D_{3} . D_{3}$ will always be reverse biased since its anode is at ground while a positive voltage is at its cathode and can be regarded as opencircuited. This makes the rectifying circuit operate as a VD.

Fig. 2 shows the voltage waveforms of the rectifying circuit in different rectifier topologies. The $\mathrm{PEH}$ is represented by its voltage source equivalent circuit model [19]. It is assumed that the diodes are ideal and the rectified voltage $V_{\text {rect }}$ is equal to $\left|v_{\mathrm{g}}\right|$ in both cases. The subscripts 'FB' and 'VD' are used to

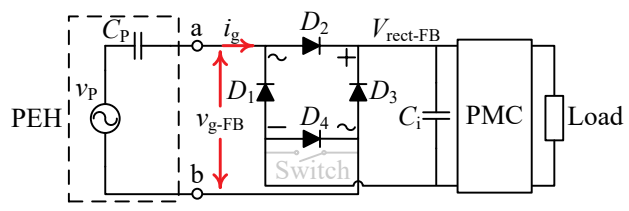

(a)



(b)

Fig. 1. Operations of the proposed rectifying circuit as a (a) FB rectifier when the switch is open and (b) a VD when the switch is closed. Greyed out components indicate that they are not conduction path. differentiate the voltages in the different rectifier topologies. It is also assumed that the PMC is not operating, which presents itself as an open circuit to the $\mathrm{PEH}$ that is experiencing a steady vibration. Thus, $v_{\mathrm{g}-\mathrm{FB}}$ across the terminals of the $\mathrm{PEH}$ that is connected to a FB rectifier is equal to $v_{\mathrm{P}}$ at steady state.

When the PEH is connected to a VD, diode $D_{2}$ blocks the current path of the PEH from terminal $b$ to terminal $a$ through external circuits such as the PMC during the negative cycle. $D_{1}$ is the only path and hence, the inherent capacitor $C_{\mathrm{P}}$ of the $\mathrm{PEH}$ will be charged up to a voltage level of $\left|v_{\mathrm{P}}\right|$. During the positive cycle, $v_{\mathrm{P}}$ and the voltage across $C_{\mathrm{P}}$ sum up as the voltage $v_{\mathrm{g}-\mathrm{VD}}$ across the $\mathrm{PEH}$, which is $2\left|v_{\mathrm{p}}\right|$ or twice $v_{\mathrm{g}-\mathrm{FB}}$. This means $V_{\text {rect-VD }}$ of a PEH that is connected to a VD is twice $V_{\text {rect-FB }}$ of a PEH that is connected to a FB rectifier under the same test condition.

\section{B. Rectifier Topologies Switching}

VD topology is used when the voltage amplitude of the $\mathrm{PEH}$ is low. When the voltage is high, the rectifying circuit switches to the FB rectifier topology. The switching of the topologies can be determined by two different threshold voltages as illustrated in Fig. 2. Assume that the rectifying circuit is currently in the VD topology, it switches to FB rectifier when $V_{\text {rect-VD }}$ exceeds a high threshold voltage $V_{\mathrm{TH}-\mathrm{VD}}$ to prevent $V_{\text {rect }}$ from becoming too high which exceeds the maximum operating voltage of the PMC due to the voltage amplification by the VD. When it is in the $\mathrm{FB}$ rectifier topology, another threshold voltage $V_{\mathrm{TH}-\mathrm{FB}}$ that is lower than $V_{\mathrm{TH}-\mathrm{VD}}$ is used. The rectifying circuit switches to VD when $V_{\text {rect-FB }}$ is lower than $V_{\text {TH-FB }}$ so that the low piezoelectric voltage can be amplified for the operation of the PMC at low voltage which is not viable with the FB rectifier.

The significance of having two threshold voltages can be explained by using the following scenario where only one threshold voltage is used, for example, only $V_{\text {TH-VD. }}$ It should be noted that when the rectifying circuit is in the VD topology, $\left|v_{\mathrm{g}-\mathrm{VD}}\right|$ is about twice $\left|v_{\mathrm{P}}\right|$ generated by the $\mathrm{PEH}$ under a given vibration excitation. When $\left|v_{\mathrm{g}-\mathrm{VD}}\right|$ is sufficiently high, $V_{\text {rect-VD }}$ exceeds $V_{\mathrm{TH}-\mathrm{VD}}$ and the rectifying circuit switches to FB rectifier. The rectified voltage will now become $V_{\text {rect-FB, which }}$ is half of $V_{\text {rect-VD }}$ where the maximum possible amplitude is $\left|v_{\mathrm{P}}\right|$. If the threshold voltage is fixed as $V_{\mathrm{TH}-\mathrm{VD}}$ and assume that $\left|v_{\mathrm{P}}\right|$ generated by the $\mathrm{PEH}$ remains the same, the rectified

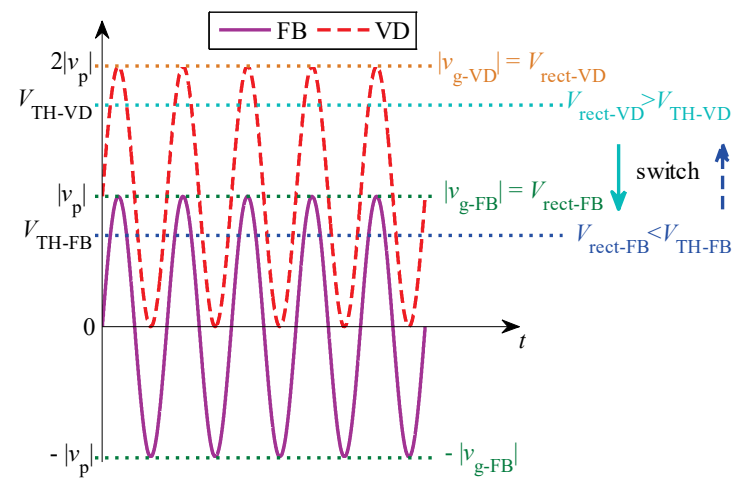

Fig. 2. Illustration of the voltage $v_{\mathrm{g}}, V_{\text {rect, }}$, and $V_{\mathrm{TH}}$ for the switching of the rectifier topologies that is connected to a FB rectifier and a VD, respectively under no load condition. 
voltage using the FB rectifier will become well below the threshold voltage. The rectifying circuit will then switch back to VD that causes the rectified voltage to exceed the threshold voltage once more, and consequently switch back to the FB rectifier again. The rectifying circuit will switch back and forth between the VD and FB rectifier topologies continuously as long as $\left|v_{\mathrm{g}}\right|$ in the VD topology is higher and $\left|v_{\mathrm{g}}\right|$ in the FB rectifier topology is lower than the threshold voltage, respectively. Such a repercussion might reduce the overall system efficiency. Therefore, two different threshold voltages for the two different topologies are needed to avoid the aforementioned problem.

\section{SYSTEM DESIGN AND ANALYSIS}

As discussed earlier, a rectifier that configures itself between the FB rectifier and VD topologies can be realized by adding a switch to a FB rectifier. A PMC as reported in [11], [24] will be used as an example to demonstrate the ease of integration of this configurable rectifier into existing PMC. The voltages $V_{\mathrm{RD}}, V_{\mathrm{BG}}$, and $V_{\mathrm{DCC}}$ are existing nodes from the control circuit of the PMC [25]. Other reported PMCs may be used as these voltages are common, although with different labels [12], [15], [26]. $V_{\mathrm{RD}}$ is $0.0641 V_{\text {rect }}$ [25], $V_{\mathrm{BG}}$ is a $1.182 \mathrm{~V}$ bandgap reference voltage, and $V_{\mathrm{DCC}}$ is an internal power rail from the PMC. $V_{\text {DCC }}$ follows $V_{\text {rect }}$ and becomes steady at 4.15 $\mathrm{V}$ after $V_{\text {rect }}$ reaches $4.6 \mathrm{~V}$ or higher. Fig. 3 shows the schematic of the configurable rectifier that consists of a FB rectifier and a switch, and its control circuit that consists of a comparator, a switch, and some resistors.

\section{A. Threshold Voltages for the Topology Switching}

The choice of $V_{\mathrm{TH}-\mathrm{FB}, \mathrm{VD}}$ has to take several factors into consideration. First is the voltage ratio $\eta_{\mathrm{V}}$ as given by (1):

$$
\eta_{\mathrm{V}}=\frac{V_{\text {rect }}}{\left|v_{\mathrm{g}}\right|-n V_{\mathrm{F}}}=\frac{\left|v_{\mathrm{g}}\right|-2 V_{\mathrm{F}}}{\left|v_{\mathrm{g}}\right|-n V_{\mathrm{F}}}, n= \begin{cases}0, & \text { for } \mathrm{FB} \\ 1, & \text { for } \mathrm{VD}\end{cases}
$$

As the vibration excitation on the PEH increases, $\left|v_{\mathrm{P}}\right|$ and $\left|i_{\mathrm{g}}\right|$ increase [6], [22]. This causes the rectifying circuit to switch from VD to FB rectifier, with $\left|v_{\mathrm{g}}\right|$ reduced by half as explained earlier. From the Shockley equation $V_{\mathrm{F}}=n V_{\mathrm{t}} \ln \left(I_{\mathrm{D}} / I_{\mathrm{S}}+1\right)$ [27], $V_{\mathrm{F}}$ slightly raises with the current through the diode $I_{\mathrm{D}}$, which is $\left|i_{\mathrm{g}}\right|$ here. From (1), $\eta_{\mathrm{V}}$ further reduces as $n V_{\mathrm{F}}$ which is a slight de offset in VD due to the charging of $C_{\mathrm{p}}$ via $D_{1}$ is zero in FB rectifier. Thus, $V_{\text {TH-FB }}$ which is also the minimum $V_{\text {rect }}$ for the circuit to operate as a FB rectifier has to be as high as possible to maintain a high $\eta_{\mathrm{V}}$ following the topology switch from VD. Aiming at $\eta_{\mathrm{V}}$ of over 0.9 for FB rectifiers is reasonable as it has been achieved by some reported rectifiers such as in [14], [17].

$V_{\mathrm{TH}-\mathrm{VD}}$ has to be higher than $2 V_{\mathrm{TH}-\mathrm{FB}}$ so that the reduction in
$V_{\text {rect }}$ as the circuit switches from VD to FB rectifier does not fall below $V_{\mathrm{TH}-\mathrm{FB}}$, which will trigger the circuit to switch back to VD. Operation at or exceeding the maximum operating voltage limit of the PMC $V_{\mathrm{PMC}(\max )}$ for extended periods will damage or affect the reliability of the PMC [28]. Thus, $V_{\mathrm{TH}-\mathrm{VD}}$ has to be lower than $V_{\mathrm{PMC}(\max )}$ so that the rectifying circuit can switch to the FB rectifier which lowers $V_{\text {rect }}$ before $V_{\text {rect }}$

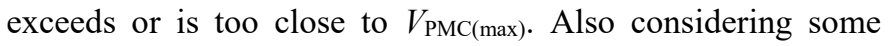
possible variations in components, a safety margin of $10 \%$ is usually adopted [29]. Therefore, $V_{\mathrm{TH}-\mathrm{FB}, \mathrm{VD}}$ need to satisfy the condition shown in (2):

$$
2 V_{\mathrm{TH}-\mathrm{FB}}<V_{\mathrm{TH}-\mathrm{VD}}<0.9 V_{\mathrm{PMC}(\max )}
$$

\section{B. Reference Voltages for Different Topologies}

The threshold voltages are represented by the reference voltages in the control circuit. The reference voltage $V_{\mathrm{RH}}$ is derived from $V_{\mathrm{BG}}$ by using a resistive divider network. Since two threshold voltages are required, two different values of reference voltages $V_{\mathrm{RH}-\mathrm{VD}}$ and $V_{\mathrm{RH}-\mathrm{FB}}$ are used. Based on (2), $V_{\mathrm{RH}-\mathrm{VD}}$ is a larger value that is used when the rectifying circuit is operating as $\mathrm{VD}$ whereas $V_{\mathrm{RH}-\mathrm{FB}}$ is less than half of $V_{\mathrm{RH}-\mathrm{VD}}$ for the circuit operation in the FB rectifier topology since $V_{\text {rect }}$ will be reduced by half when the circuit switches from VD to FB rectifier. $V_{\text {RH-VD }}$ and $V_{\text {RH-FB }}$ are obtained by turning the $\mathrm{N}-$ type MOSFET $M_{\text {ref }}$ on or off as given by (3):

$$
V_{\mathrm{RH}}= \begin{cases}V_{\mathrm{RH}-\mathrm{VD}}, & \text { for } M_{\text {ref }} \text { is on } \\ V_{\mathrm{RH}-\mathrm{FB}}, & \text { for } M_{\text {ref }} \text { is off }\end{cases}
$$

When $M_{\text {ref }}$ is switched on, it acts as a closed switch that shorts the resistor $R_{\mathrm{H} 1}$. Thus, $V_{\mathrm{RH}-\mathrm{VD}}$ is expressed as (4):

$$
V_{\mathrm{RH}-\mathrm{VD}}=\frac{R_{\mathrm{H} 3}}{R_{\mathrm{H} 2}+R_{\mathrm{H} 3}} V_{\mathrm{BG}}
$$

When $M_{\text {ref }}$ is switched off, it is an open circuit that does not have any effect on the resistive divider network. Thus, $V_{\mathrm{RH}-\mathrm{FB}}$ is expressed as (5):

$$
V_{\mathrm{RH}-\mathrm{FB}}=\frac{R_{\mathrm{H} 3}}{R_{\mathrm{H} 1}+R_{\mathrm{H} 2}+R_{\mathrm{H} 3}} V_{\mathrm{BG}}
$$

Equations (4) and (5) are almost identical apart from their denominators where (4) is the sum of $R_{\mathrm{H} 2}$ and $R_{\mathrm{H} 3}$ whereas (5) is the sum of $R_{\mathrm{H} 1}, R_{\mathrm{H} 2}$, and $R_{\mathrm{H} 3}$. Therefore, $V_{\mathrm{RH}-\mathrm{FB}}$ is lower than $V_{\mathrm{RH}-\mathrm{VD}}$ because of its larger denominator that includes $R_{\mathrm{H} 1}$.

\section{Switch for Rectifying Circuit}

The switch $M_{\text {rect }}$ is realized by using an N-type MOSFET. The SOURCE and DRAIN of $M_{\text {rect }}$ are connected to the anode and cathode of diode $D_{4}$, respectively. This ensures that the inherent body diode of $M_{\text {rect }}$ is in the same direction of $D_{4}$ without creating a conduction path in the reverse direction of

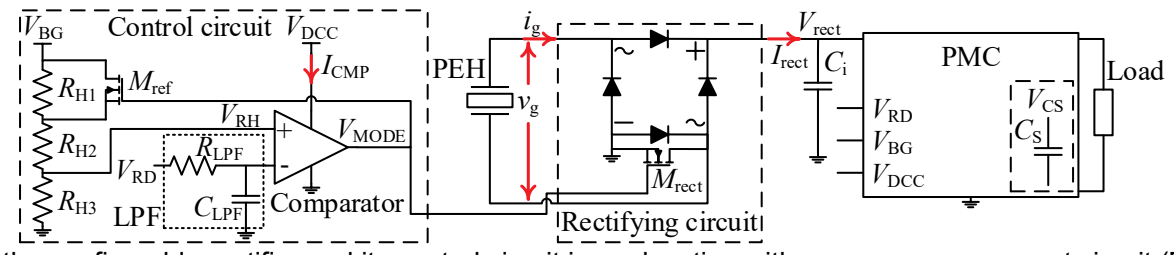

Fig. 3. Circuit diagram of the configurable rectifier and its control circuit in conjunction with a power management circuit (PMC). 
$D_{4}$. Given that the SOURCE of $M_{\text {rect }}$ is referenced to the ground of the PMC, turning on or off $M_{\text {rect }}$ is straight forward.

When the voltage applied at the GATE of $M_{\text {rect }}$ is $0 \mathrm{~V}$ or lower than the threshold voltage of the MOSFET, $M_{\text {rect }}$ is turned off and acting as an opened switch. Therefore, the rectifying circuit is operating as a FB rectifier. $M_{\text {rect }}$ is turned on when a voltage that is higher than the threshold voltage of the MOSFET is applied at its GATE. Once $M_{\text {rect }}$ is turned on, it bypasses $D_{4}$ and consequently turns the FB rectifier into a VD.

\section{Switching Control}

The comparator is the main active device in the control circuit. It compares $V_{\mathrm{RD}}$ with $V_{\mathrm{RH}}$ to determine whether the amplitude of the voltage across the terminals of the PEH has exceeded the threshold voltages. It then turns on or off $M_{\text {rect }}$ and $M_{\text {ref }}$ to achieve the reconfiguration of the rectifier topology and the change of the value of the reference voltage. Switching on $M_{\text {rect }}$ and $M_{\text {ref }}$ leads to the configuration as the VD topology and $V_{\mathrm{RH}}$ with the value of $V_{\mathrm{RH}-\mathrm{VD}}$. On the contrary, the configuration that uses the FB rectifier topology with the reference voltage $V_{\text {RH-FB }}$ requires $M_{\text {rect }}$ and $M_{\text {ref }}$ to be switched off. The operation of the control circuit is explained as follows.

$V_{\mathrm{RD}}$ and $V_{\mathrm{RH}}$ are fed to the negative input and the positive input of the comparator, respectively. $V_{\mathrm{BG}}$ follows the input voltage into the PMC, which in this case is $V_{\text {rect }}$ until it reaches $1.182 \mathrm{~V}$. Given that $V_{\mathrm{RD}}$ is the scaled down voltage of $V_{\text {rect }}$, $V_{\mathrm{RD}}$ can be assumed to be lower than $V_{\mathrm{RH}}$ initially. The output of the comparator $V_{\text {MODE }}$ becomes HIGH to turn on both $M_{\text {rect }}$ and $M_{\text {ref. }}$ Therefore, the rectifying circuit is now operating using the VD topology and the reference voltage is $V_{\mathrm{RH}-\mathrm{VD}}$. When $V_{\mathrm{RD}}$ exceeds $V_{\mathrm{RH}-\mathrm{VD}}$ due to high vibration excitation on the PEH, $V_{\text {MODE }}$ becomes LOW, which turns off both $M_{\text {rect }}$ and $M_{\text {ref. }}$. The rectifying circuit subsequently operates as a FB rectifier with a lower reference voltage value of $V_{\mathrm{RH}-\mathrm{FB}}$.

It should be noted that there will be some ripples at $V_{\text {rect }}$ due to low vibration frequencies of the $\mathrm{PEH}$, small smoothing capacitor, and energy transfer process from the PEH to the storage capacitor via the PMC [30]. Although the ripple can be reduced by increasing the capacitance $C_{\mathrm{i}}$, it is preferable to keep it small for a faster settling time for maximum power point tracking [26]. Therefore, $V_{\mathrm{RD}}$ which is scaled down from $V_{\text {rect }}$ will have some ripples too. This may cause the circuit to

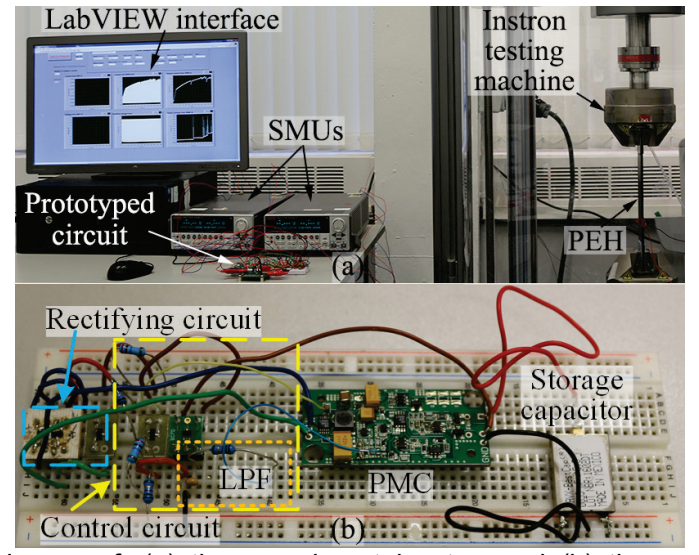

Fig. 4. Image of: (a) the experimental setup and (b) the prototyped falsely switch its rectifier topology. To mitigate this, $V_{\mathrm{RD}}$ goes through an $R C$ low-pass filter (LPF) before being fed to the comparator.

\section{EXPERIMENTAL VERIFICATION}

\section{A. Experimental Setup}

A commercially available macro fiber composite (MFC) M8528-P2 from Smart Material was bonded onto a piece of carbon fiber composite material as the PEH [11]. An Instron E10000 ElectroPuls dynamic testing machine was used to apply different strain loadings at different frequencies onto the PEH. The voltages and currents shown in Fig. 3 were measured using the Keithley 2612B sourcemeters (SMUs) which were controlled by a LabVIEW interface as shown in Fig. 4(a).

\section{B. Prototype Implementation}

Due to the simplicity of the proposed circuit and for the ease of measurement, a prototype was built using commercially available discrete components on a breadboard as shown in Fig. 4(b). The comparator used is TLV3691 for its low power consumption and low minimum operating voltage of $0.9 \mathrm{~V}$. The chosen MOSFET for $M_{\text {rect }}$ and $M_{\text {ref }}$ is BSS816NW for its low DRAIN-SOURCE resistance of 107 $\mathrm{m} \Omega$ and GATE threshold voltage of $0.55 \mathrm{~V}$. BAS70 Schottky diode is used as $D_{1}-D_{4}$ for its low typical $V_{\mathrm{F}}$ of $0.3 \mathrm{~V}$ and low reverse current. The chosen components have very stable performances against temperature variations. With very few components in the circuit, the risk of a large behavioral change due to the effects of temperature variations and noise such as thermal noise from the components are negligible under normal operating conditions [6].

The choice of the threshold voltages is based on (1) and (2), and for the ease of the circuit implementation in terms of the selection for the values of the resistors $R_{\mathrm{H} 1}, R_{\mathrm{H} 2}$, and $R_{\mathrm{H} 3}$. The PMC used has a $V_{\mathrm{PMC}(\max )}$ of $20 \mathrm{~V}$ [28]. Therefore, the rectifying circuit was set to switch from the VD topology to the FB rectifier topology when $V_{\text {rect }}$ reaches $15.4 \mathrm{~V}$ to prevent $V_{\text {rect }}$ from exceeding the voltage limit of the PMC due to the voltage amplification by the VD as the vibration continues to increase. $R_{\mathrm{H} 1}, \quad R_{\mathrm{H} 2}$, and $R_{\mathrm{H} 3}$ are $120 \quad \mathrm{M} \Omega, \quad 20 \mathrm{M} \Omega$ (VR25000002005KA1), and $100 \mathrm{M} \Omega$ (RGP0207CHJ100M), respectively. The large resistances were chosen to minimize the power dissipated by the resistors. Resistors with low tolerances and the same temperature coefficient are preferred to minimize the deviation range of the resistances from their nominal values. With the chosen resistors, the rectifying circuit will switch to VD at $V_{\text {rect }}$ of $7.68 \mathrm{~V}$, which satisfies the conditions in (1) and (2) with $V_{\mathrm{F}}$ of up to $0.4 \mathrm{~V}$. If the proposed circuit was implemented all by using surface mount components including 0603 case size resistors, it will occupy an area of around $16 \mathrm{~mm}^{2}$.

\section{Testing Method}

Various low peak-to-peak strain loadings at $10 \mathrm{~Hz}$ were applied onto the PEH so that $\nu_{\mathrm{P}}$ is low to determine the minimum start-up voltage of the prototyped circuit. Then, for 
the purpose of comparison, the same strain loading will be applied onto the PEH which is connected to a FB rectifier.

The proposed circuit will operate as a FB rectifier when the voltage from the $\mathrm{PEH}$ is high. However, a direct comparison of the proposed circuit with a circuit that uses a fixed VD will not be carried out to avoid applying high voltage stress onto the PMC when there is a high vibration excitation on the PEH.

A sweep test of varying peak-to-peak strain loadings from 0 to $500 \mu \varepsilon$ at $10 \mathrm{~Hz}$ applied onto the $\mathrm{PEH}$ which is connected to the proposed circuit was carried out to determine the response of the circuit to real-time changes. To validate the circuit design, the operation of the proposed circuit was compared with a similar circuit without the LPF and a circuit that uses only one reference voltage without the reference voltage switching.

The power consumption of the proposed control circuit was determined from the measurements of $V_{\mathrm{DCC}}$ and $I_{\mathrm{CMP}}$. The power dissipated by the resistive divider network of $R_{\mathrm{H}}$ was calculated using $V_{\mathrm{BG}}^{2} / R_{\mathrm{H}}$, where $R_{\mathrm{H}}$ is either the denominator of (4) or (5) depending on the circuit operation. The average power dissipated by the diode rectifier $P_{\mathrm{D}}$ was determined by (6):

$$
P_{\mathrm{D}}=\frac{1}{T} \int_{0}^{T} v_{\mathrm{g}}(t) i_{\mathrm{g}}(t) \mathrm{d} t-\frac{1}{T} \int_{0}^{T} V_{\text {rect }}(t) I_{\text {rect }}(t) \mathrm{d} t
$$

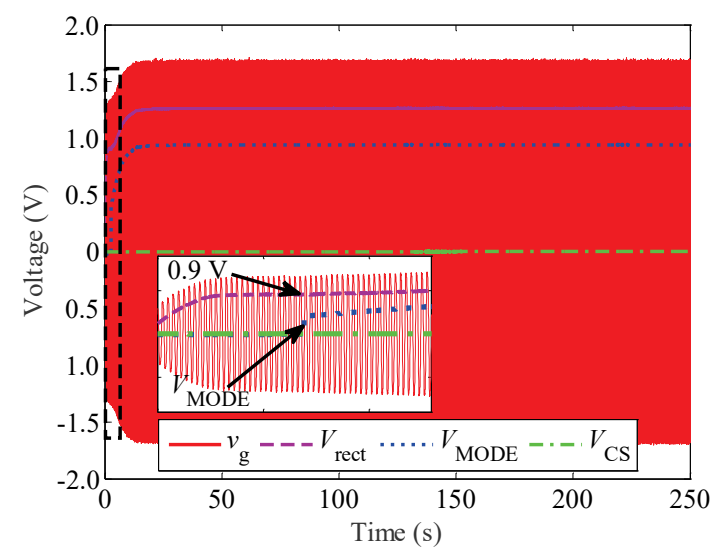

Fig. 5. Measurements from a circuit that is connected to a $\mathrm{PEH}$ via a FB rectifier. The area within the dashed line is enlarged in the inset.

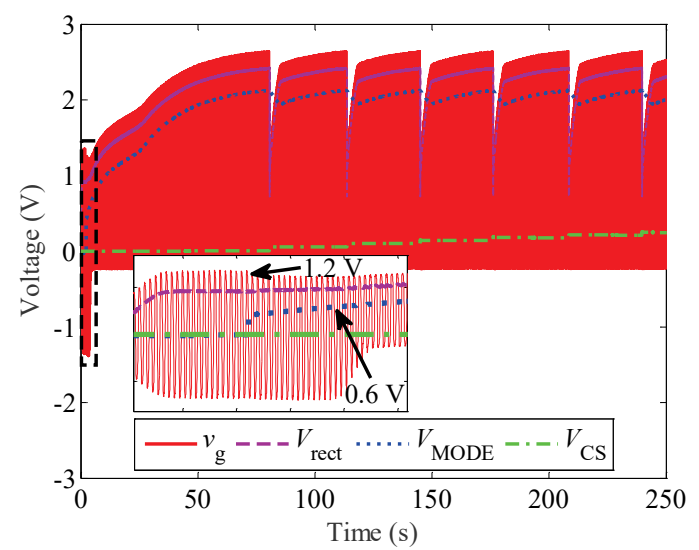

Fig. 6. Measurements from a circuit that is connected to a PEH via the proposed rectifying circuit. The area within the dashed line is enlarged in the inset.

\section{RESUlts AND Discussions}

\section{A. Start-up of the Proposed Rectifying Circuit}

A peak-to-peak strain loading of $25 \mu \varepsilon$ at $10 \mathrm{~Hz}$ was found to be the minimum vibration excitation applied onto the $\mathrm{PEH}$ that can power up the entire circuit including the PMC. It should be noted that lower strain loadings applied onto the $\mathrm{PEH}$ are still able to power up the rectifying circuit and its control circuit but not the PMC and hence, are not considered as the minimum start-up condition in this work.

Fig. 5 shows the measured $v_{\mathrm{g}}, V_{\text {rect, }} V_{\mathrm{MODE}}$, and voltage $V_{\mathrm{CS}}$ across the storage capacitor of the PMC with a FB rectifier. It was done by deliberately removing $M_{\text {rect }}$ from the proposed rectifier so that it was unable to switch to VD. $v_{\mathrm{g}}$ from the $\mathrm{PEH}$ reaches a maximum amplitude of $1.7 \mathrm{~V}$, which is too low for the PMC which uses a buck converter with a minimum operating voltage of $2.7 \mathrm{~V}$ to operate [28]. Therefore, no energy can be transferred from the PEH to the storage capacitor, where $V_{\text {rect }}$ remains steady at $1.3 \mathrm{~V}$ and $V_{\mathrm{CS}}$ is $0 \mathrm{~V}$. However, from the inset of Fig. 5, the rectifying circuit is able to operate where $V_{\text {MODE }}$ becomes $\mathrm{HIGH}$ when $V_{\text {rect }}$ is $0.9 \mathrm{~V}$.

Using the proposed circuit under the same strain loading condition, $v_{\mathrm{g}}$ can be amplified to more than $1.7 \mathrm{~V}$ as shown in Fig. 6. Energy transfer occurs when $V_{\text {rect }}$ reaches the minimum input operating voltage of the buck converter. $V_{\text {rect }}$ drops while $V_{\text {CS }}$ increases following the energy transfer. The average output power from the PEH is $3.96 \mu \mathrm{W}$. From cold start where

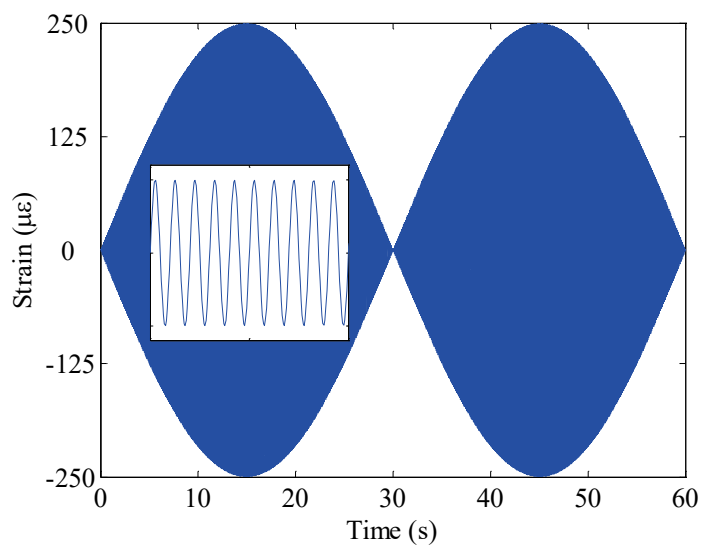

Fig. 7. Varying strain loading profile applied onto the $\mathrm{PEH}$ which is connected to the proposed circuit. The inset shows the detail of the waveform.

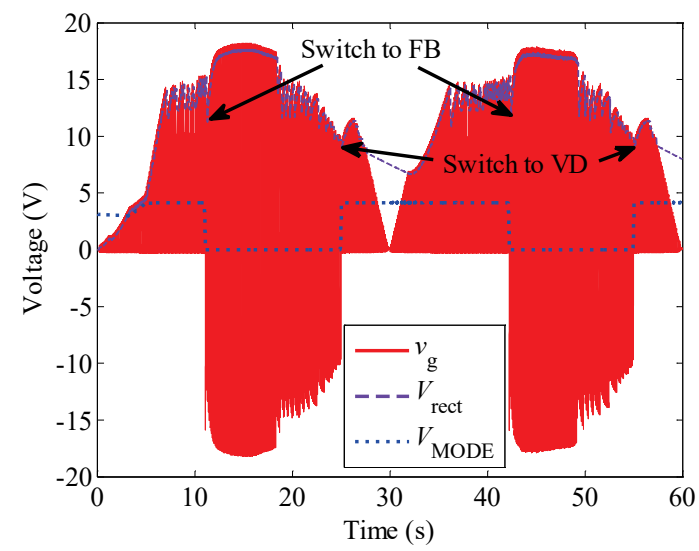

Fig. 8. Measured $v_{g}, V_{\text {rect, }}$ and $V_{\text {MODE }}$ from the proposed circuit which correspond to the applied strain profile shown in Fig. 7 . 
all the capacitors within the PMC are fully discharged, the proposed circuit operates as a FB rectifier initially because the voltage from the PEH is lower than the minimum operating voltage of the control circuit. Similar to the inset of Fig. 5, the inset of Fig. 6 shows the proposed circuit can start up once $v_{\mathrm{g}}$ reaches $1.2 \mathrm{~V}$, where $V_{\text {rect }}$ and hence $V_{\text {DCC }}$ becomes $0.9 \mathrm{~V}$ due to the diode forward voltage drop. $V_{\text {MODE }}$ becomes HIGH at about $0.4 \mathrm{~V}$ initially and gradually increases as more energy is accumulated. The proposed circuit switches to the VD topology when $V_{\text {MODE }}$ reaches $0.6 \mathrm{~V}$, which is the threshold voltage to turn on $M_{\text {rect. }}$ Therefore, the proposed rectifying circuit can start up with a minimum voltage of $1.2 \mathrm{~V}$ from a $\mathrm{PEH}$, which is similar to some reported circuits that use an active rectifier [14], [15]. If there are some residual charges in the capacitors of the PMC that are sufficient for the operation of the proposed rectifying circuit, the operation will begin with the VD topology straightaway even when the piezoelectric voltage is lower than $1.2 \mathrm{~V}$.

\section{B. Validation of the Circuit Operation}

Fig. 7 shows a varying strain loading profile that was used for the sweep test. The peak-to-peak strain level varies from 0 to $500 \mu \varepsilon$ and then back to $0 \mu \varepsilon$ for $30 \mathrm{~s}$ twice. Fig. 8 shows the corresponding $v_{\mathrm{g}}, V_{\text {rect }}$, and $V_{\text {MODE }}$ measured as the strain profile shown in Fig. 7 was applied onto the PEH which was connected to the proposed circuit. The proposed circuit operates as expected where it begins with the VD topology

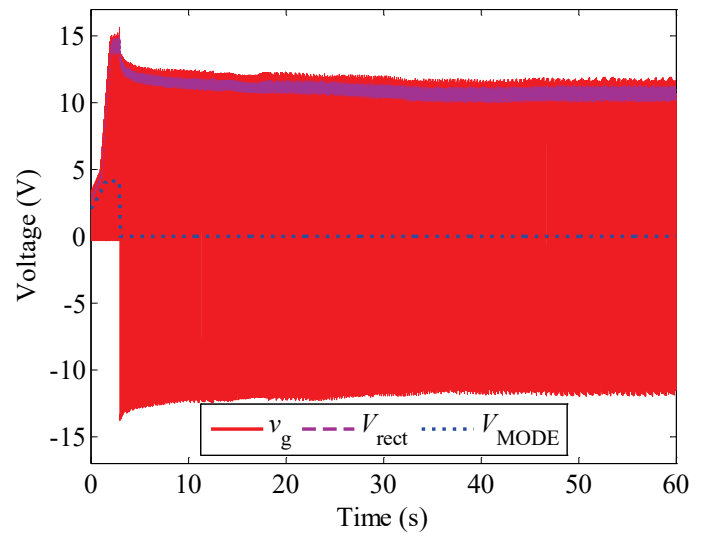

Fig. 9. Measured voltages at the proposed circuit when the connected $\mathrm{PEH}$ was subjected to a peak-to-peak strain level of $300 \mu \varepsilon$ at $10 \mathrm{~Hz}$.



Fig. 10. Measured voltages at the circuit without the LPF when the connected PEH was subjected to a peak-to-peak strain level of $300 \mu \varepsilon$ at $10 \mathrm{~Hz}$. The area within the dashed line is enlarged in the inset. when the voltage is still low. The circuit is known to be operating using the VD topology because the measured $v_{\mathrm{g}}$ does not show any waveform in the negative polarity and $V_{\text {MODE }}$ is HIGH. When $V_{\text {rect }}$ reaches $15.4 \mathrm{~V}$, the rectifying circuit switches from VD to FB rectifier. Therefore, $v_{\mathrm{g}}$ now exhibits a waveform profile with both positive and negative polarities, and $V_{\text {MODE }}$ is LOW. It should also be noted that when the rectifying circuit switches from VD to FB rectifier, there is a slight drop in both $v_{\mathrm{g}}$ and $V_{\text {rect }}$. When the rectifying circuit switches from FB rectifier to VD, there is a slight increase in both $v_{\mathrm{g}}$ and $V_{\text {rect. }}$. This is expected as explained in Section II.A where VDs increase the voltage from the PEH whereas FB rectifiers do not.

\section{Validation of the Proposed Control Mechanism}

In the test that determines the need to have the LPF and the different reference voltages instead of a fixed one, the PEH was tested at a peak-to-peak strain loading of $300 \mu \varepsilon$ at $10 \mathrm{~Hz}$ as an example. Fig. 9 shows the same measurements on the proposed circuit as in the sweep test. The circuit operates as a VD at the beginning and switches to FB rectifier when the threshold voltage is reached, as can be seen from the change of waveform in $v_{\mathrm{g}}$ and the state of $V_{\text {MODE }}$. Without the voltage amplification by the VD as explained in Section II.A, $\left|v_{\mathrm{g}}\right|$ and $V_{\text {rect }}$ reduce after the switch from VD to FB rectifier. With the threshold voltage lowered as well, the rectifying circuit

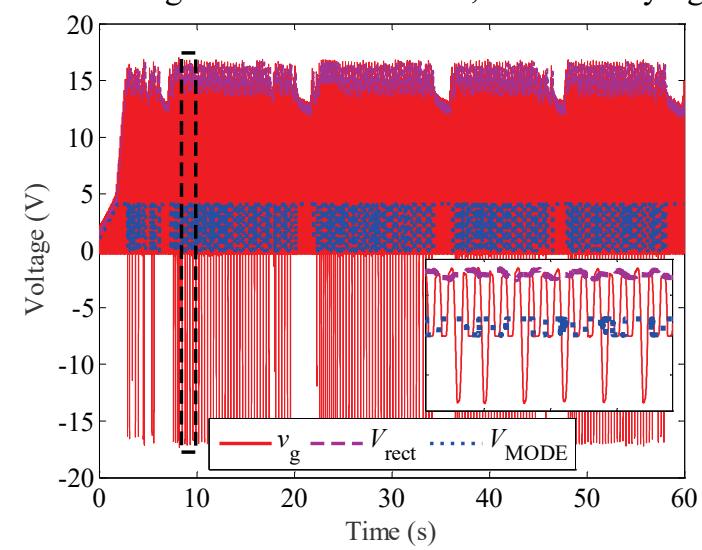

Fig. 11. Measured voltages at the circuit with a fixed reference voltage when the connected PEH was subjected to a peak-to-peak strain level of $300 \mu \varepsilon$ at $10 \mathrm{~Hz}$. The area within the dashed line is enlarged in the inset.

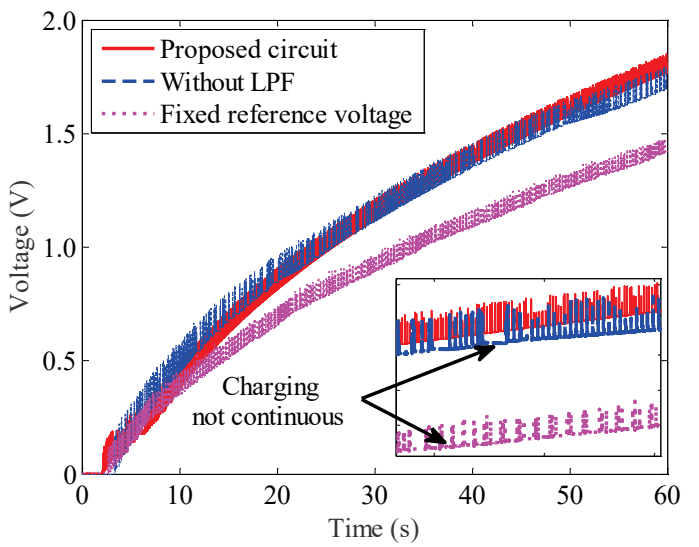

Fig. 12. Comparison of the capacitor voltage after the storage capacitor was charged up by a PMC with the proposed control circuit, the proposed circuit without LPF, and a fixed reference voltage. 


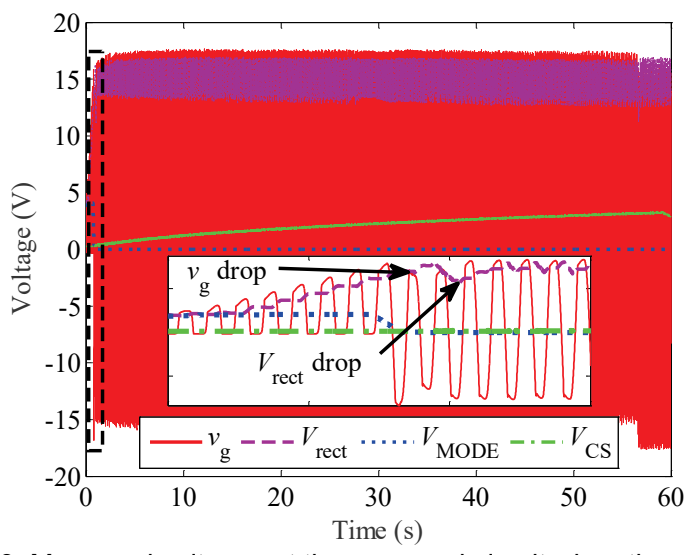

Fig. 13. Measured voltages at the proposed circuit when the connected PEH was subjected to a peak-to-peak strain level of $500 \mu \varepsilon$ at $10 \mathrm{~Hz}$. The area within the dashed line is enlarged in the inset.

remains as FB rectifier.

Fig. 10 shows the test without the LPF. The ripple of $V_{\text {rect }}$ seems to be larger because of the fast switching between the two different rectifier topologies as shown in the inset of Fig. 10. $V_{\text {MODE }}$ is short in terms of its duration and amplitude, which indicates a very brief switching from the FB rectifier topology to the VD topology and back to FB rectifier again. In another test, $R_{\mathrm{H} 1}$ and $M_{\text {ref }}$ were removed so that the terminal of $V_{\mathrm{BG}}$ connects directly to $R_{\mathrm{H} 2}$ to make the circuit to have only one fixed reference voltage of $V_{\mathrm{RH}-\mathrm{VD}}$ without the reference voltage switching capability. Fig. 11 shows that with only one fixed reference voltage, the circuit switches back and forth between the two different rectifier topologies continuously as shown in the inset of Fig. 11 where $V_{\text {MODE }}$ can be seen to have a wider duration to allow a complete switching of the rectifier topologies. The effect of such an undesirable switching was examined by measuring the capacitor voltage $V_{\mathrm{CS}}$.

Fig. 12 shows the comparison of $V_{\mathrm{CS}}$ of a PMC with the proposed rectifying circuit, the control circuit without LPF, and the circuit with a fixed reference voltage in charging up a $22 \mathrm{mF}$ storage capacitor. After $60 \mathrm{~s}$ of charging, $V_{\mathrm{CS}}$ of the proposed circuit is higher than the circuit with a fixed reference voltage by $20 \%$. This shows that without the proposed reference voltage switching, the continuous switching between the two rectifier topologies distorted the charging of the storage capacitor and deteriorates the amount of harvested energy. This is because the voltage for maximum power transfer changes as the rectifier topology changes despite the fixed piezoelectric voltage [19]. However, the switching of rectifier topologies keeps repeating at the same threshold voltage. Therefore, $V_{\text {rect }}$ as well as $v_{\mathrm{g}}$ are held at a relatively constant level that is away from the optimum voltage for maximum power transfer in either rectifier topology. $V_{\mathrm{CS}}$ of the circuit without the LPF is only slightly lower than the proposed circuit because the switching of the rectifier topology is incomplete for most of the time. Thus, there is less distortion to the optimum voltage level for maximum power transfer. It should be noted that the charging of $C_{\mathrm{S}}$ is not continuous due to the distortion in both cases without the proposed method as shown in the inset of Fig. 12. This also increases the leakage from $C_{\mathrm{S}}$ and leads to the lower

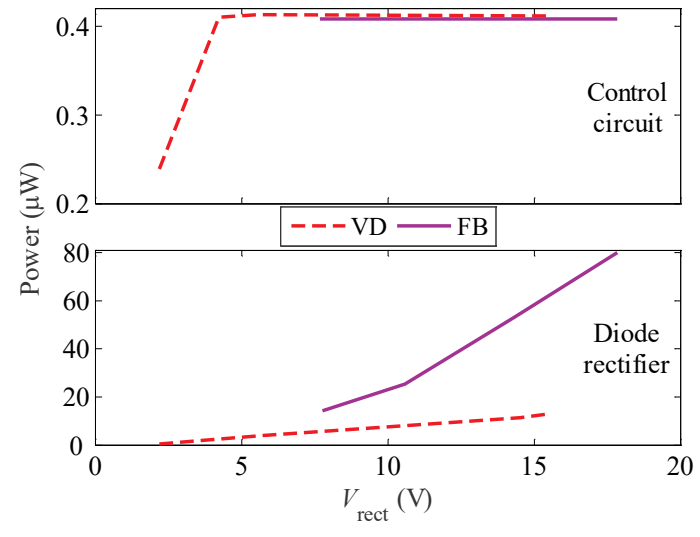

Fig. 14. Power consumption and dissipation of the proposed rectifying circuit in different rectifier topologies against $V_{\text {rect }}$.

$V_{\text {CS. }}$

Fig. 13 shows the measured voltages of $v_{\mathrm{g}}, V_{\text {rect, }}, V_{\mathrm{MODE}}$, and $V_{\mathrm{CS}}$ at the proposed circuit when the PEH was subjected to a peak-to-peak strain loading of $500 \mu \varepsilon$ at $10 \mathrm{~Hz}$. The inset shows that there will always be a slight drop in the voltage across the PEH when the rectifying switches from the VD topology to the FB rectifier topology. This is regardless of whether the voltage $v_{\mathrm{g}}$ from the PEH using the FB rectifier is eventually higher or lower than the triggering point of $V_{\mathrm{TH}-\mathrm{VD}}$ as in the case shown in Fig. 9. This further proves the need for the reference voltage switching to avoid unnecessary switching back to the previous rectifier topology. It should be noted $V_{\text {rect }}$ does not drop immediately because the $C_{\mathrm{i}}$ still holds some charges.

Also, with the same $22 \mathrm{mF}$ storage capacitor, $V_{\mathrm{CS}}$ in Fig. 13 reaches $3.3 \mathrm{~V}$ in less than $60 \mathrm{~s}$. Comparing with the maximum $V_{C S}$ in Fig. 12 that was charged up to around $1.8 \mathrm{~V}$ when the peak-to-peak strain loading applied onto the PEH was $300 \mu \varepsilon$, it is clear that more power can be obtained from the PEH if the vibration excitation is higher. This result shows the rationale of using FB rectifier when the voltage is high. If a fixed VD was used when the PEH in this work was subjected to a peakto-peak strain level of $300 \mu \varepsilon, V_{\text {rect }}$ would have reached $20 \mathrm{~V}$, which is the maximum voltage limit of the PMC used in the experiment. Applying higher strain levels onto the PEH would be prohibitive as it will cause $V_{\text {rect }}$ to exceed the maximum voltage limit of the PMC, where the PMC could be permanently damaged and may no longer functioning properly. This means a PMC with a fixed VD for rectification has a lower upper limit of the power transfer range due to the voltage amplification by the VD that may cause $V_{\text {rect }}$ to exceed the $V_{\mathrm{PMC}(\max )}$ of the PMC even when the vibration excitations on the PEH is low.

\section{Power Consumption, Dissipation, and Loss}

Fig. 14 shows the power consumption of the control circuit and dissipation in the rectifier of the proposed rectifying circuit against the rectified voltage. The proposed rectifying circuit operates using the VD topology when the piezoelectric voltage is low up until the threshold voltage of $V_{\mathrm{TH}-\mathrm{VD}}$. The rectifying circuit operates using the FB rectifier topology only when the piezoelectric voltage is higher than the preset 
TABLE I

COMPARISON WITH OTHER SOLUTIONS

\begin{tabular}{|c|c|c|c|c|}
\hline Reference & Technique & $\begin{array}{l}\text { Piezoelectric } \\
\text { Transducer }\end{array}$ & $\eta_{\mathrm{P}}$ & $\begin{array}{l}\text { Voltage } \\
\text { range }\end{array}$ \\
\hline [14] & $\begin{array}{l}\text { Active } \mathrm{FB} \\
\text { rectifier }\end{array}$ & $\begin{array}{l}\text { Emulated with } \\
\text { voltage source }\end{array}$ & $\begin{array}{l}0.90 \\
\text { (peak) }\end{array}$ & $\mathrm{N} / \mathrm{A}$ \\
\hline [19] & $\begin{array}{l}\text { Passive VD } \\
\text { rectifier }\end{array}$ & $\begin{array}{l}1 \text { Piezo Systems } \\
\text { Q220-A4-303YB }\end{array}$ & $>0.95$ & $3-20 \mathrm{~V}$ \\
\hline [22] & $\begin{array}{l}\text { Connection } \\
\text { switching }\end{array}$ & 2 Mide V20W & $0.10-0.50$ & $2-12 \mathrm{~V}$ \\
\hline [23] & $\begin{array}{l}\text { Split- } \\
\text { electrode }\end{array}$ & $\begin{array}{l}1 \text { custom PEH } \\
\text { with } 8 \text { electrodes }\end{array}$ & $\begin{array}{l}0.22-0.52 \\
\text { (FBR) }\end{array}$ & $\begin{array}{l}1.5-20 \\
\mathrm{~V}\end{array}$ \\
\hline $\begin{array}{l}\text { This } \\
\text { work }\end{array}$ & $\begin{array}{l}\text { Topology } \\
\text { switching }\end{array}$ & $\begin{array}{l}\text { 1 Smart Material } \\
\text { MFC8528-P2 }\end{array}$ & $0.93-0.98$ & $\begin{array}{l}1.2-20 \\
\mathrm{~V}\end{array}$ \\
\hline
\end{tabular}

threshold voltages. As the rectifying switches from VD to FB rectifier when $V_{\text {rect }}$ exceeds the threshold voltage, $V_{\text {rect }}$ decreases without the voltage amplification from the VD. With the reference voltage lowered as well, the rectifying circuit continues to operate using the FB rectifier topology as long as $V_{\text {rect }}$ is above $V_{\text {TH-FB }}$. Thus, there are two power consumptions within a certain range of $V_{\text {rect }}$ as the rectifying circuit could be operating using either topology depending on the circumstances explained.

The power consumptions are slightly different with different rectified voltage and rectifier topologies due to the difference in the voltage $V_{\text {DCC }}$ applied onto the comparator and the resistance of the resistive divider network in the different rectifier topologies used, respectively. As discussed earlier in Section III, $V_{\text {DCC }}$ follows the amplitude of the rectified voltage $V_{\text {rect }}$ until it reaches $4.6 \mathrm{~V}$. Therefore, the power consumption is as low as $240 \mathrm{nW}$ initially due to the low $V_{\text {rect }}$ and increases linearly with $V_{\text {rect. }}$ The maximum power consumption becomes relatively constants at around $410 \mathrm{nW}$ when $V_{\text {rect }}$ is higher than $4.6 \mathrm{~V}$ because $V_{\text {DCC }}$ becomes steady at around $4.15 \mathrm{~V}$.

The component that consumes the most power in the proposed control circuit is the comparator. The comparator consumes about $97 \mathrm{nA}$ of current in all the test conditions. However, the resistance of the resistive network in different rectifier topologies is different as depicted by (4) and (5). The total resistance in the resistive divider network is higher in the FB rectifier topology, which leads to a slightly lower power dissipation at $404 \mathrm{nW}$ for the FB rectifier topology. Given that the overall power consumption of the proposed rectifying circuit is low in the nanowatts range, it has very little impact on the existing PMC as shown in Fig. 5 and Fig. 6 where the circuit can start-up despite the low power from the PEH.

However, the power dissipation $P_{\mathrm{D}}$ of $\mathrm{FB}$ rectifier is much higher at $14.31-79.97 \mu \mathrm{W}$ compared with $\mathrm{VD}$ at $0.36-12.75$ $\mu \mathrm{W}$. $P_{\mathrm{D}}$ increases with $V_{\text {rect }}$ in both topologies, which is expected since the piezoelectric voltage and current usually increase with the vibration excitation applied onto the PEH. Based on the Shockley equation, $V_{\mathrm{F}}$ in FB rectifier is higher than VD because the circuit switches to FB rectifier when the piezoelectric voltage has increased to a sufficiently high level above the threshold voltage due to higher vibration excitations. This means the current generated by the $\mathrm{PEH}$ that flows through the diodes is also higher in FB rectifier. Furthermore, the numerator of (1) for VD can be seen as $\left(\left|v_{\mathrm{g}}\right|-\right.$

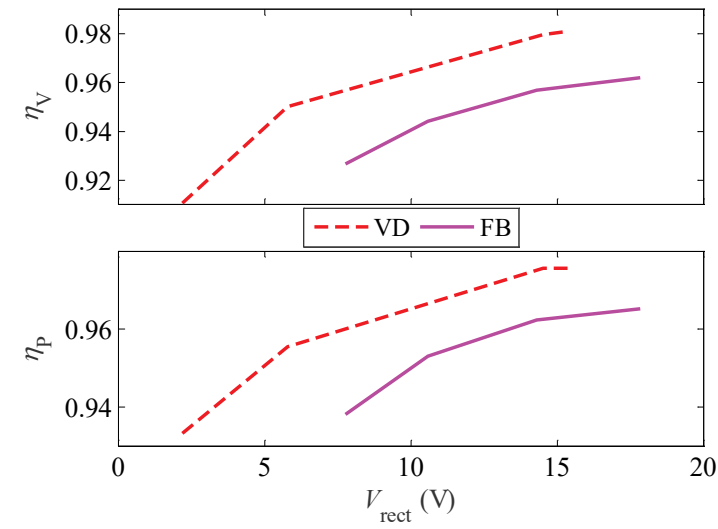

Fig. 15. Voltage and power ratios of the proposed rectifying circuit in different rectifier topologies against $V_{\text {rect. }}$

$\left.V_{\mathrm{F}}\right)-V_{\mathrm{F}}$ where the term $\left(\left|v_{\mathrm{g}}\right|-V_{\mathrm{F}}\right)$ is the voltage in the positive half cycle that will be rectified after subtracting the offset that occurs during the negative half cycle that charges $C_{\mathrm{P}}$ via $D_{1}$. Thus, only one $V_{\mathrm{F}}$ will contribute to $P_{\mathrm{D}}$ in $\mathrm{VD}$ at a time in each of the half cycles of $v_{\mathrm{g}}$ whereas $v_{\mathrm{g}}$ has to go through the diode pairs of $D_{2,4}$ or $D_{3,1}$ in FB rectifier, which is a loss of $2 V_{\mathrm{F}}$ in each of its half cycle.

\section{E. Comparison with Other Solutions}

Table I compares some features of the proposed circuit that are common with other reported solutions since a like-for-like comparison is difficult due to the different implementation technologies and operating environments. The proposed circuit does not require an additional or custom made transducer to achieve an extended operating range. The power ratio $\eta_{\mathrm{P}}$ is high at more than 0.9 and is comparable with some reported rectifiers. $\eta_{\mathrm{P}}$ is obtained by taking the second term over the first term of (6). Fig. 15 shows that $\eta_{\mathrm{P}}$ follows the same trend as $\eta_{\mathrm{V}}$ because $\eta_{\mathrm{P}}$ contains exactly the same voltage terms as $\eta_{\mathrm{V}}$ in addition to the currents $i_{\mathrm{g}}$ and $I_{\text {rect }}$ where the amplitudes of the currents are the same. $\eta_{\mathrm{V}}$ remains high above 0.9 throughout the whole circuit operation, which justified that the chosen $V_{\mathrm{TH}-\mathrm{FB}}$ for the topology switch from VD to FB rectifier is high enough although $\eta_{\mathrm{V}}$ drops following the switch, which is expected due to the higher loss in FB rectifier as explained earlier. The operation range of the proposed reconfigurable rectifier is also comparable with other solutions. However, it should be noted that the maximum operating voltage is mainly limited by the voltage limit of the PMC and MOSFET used as the diodes used can withstand a voltage of up to $70 \mathrm{~V}$. Therefore, it is possible to further extend the operation range of the system if other PMCs and components with higher voltage limits are used.

\section{CONCLUSION}

An adaptive self-configurable diode based rectifier to allow piezoelectric energy harvesting over a wider range than a circuit that uses only one fixed rectifier topology of VD or FB rectifier is presented. The proposed rectifying circuit switches between the VD and the FB rectifier topology based on the voltage from the PEH. It can be easily realized by adding a 
switch to a passive FB rectifier. The rectifying circuit operates as a VD when the switch is closed, and a FB rectifier when the switch is open. The proposed rectifying circuit was integrated into an existing PMC to demonstrate its ease of implementation and operation.

The proposed rectifying circuit is able to cold start from a piezoelectric voltage of $1.2 \mathrm{~V}$, which is comparable to other reported active rectifying circuits. The PMC with the proposed rectifying circuit can begin its operation with a much lower vibration excitation applied onto the PEH than the PMC without the proposed rectifying circuit. Low voltage from the PEH due to low vibration excitation is amplified by the rectifier using the VD topology to reach the minimum operating voltage of the PMC for energy transfer. When the voltage from the PEH exceeds a threshold voltage, the circuit switches to FB rectifier so that the voltage from the PEH is not amplified to the extent that it exceeds the maximum operating voltage of the PMC. This allows the PMC to continue harvesting energy from the $\mathrm{PEH}$ at higher vibration excitations which have a higher power.

Reference voltage switching approach is employed where two different reference voltages are used by the control circuit for the different rectifier topologies. This prevents the rectifying circuit from switching unexpectedly between the two different rectifier topologies, which affects the amount of power harvested from the PEH by the PMC. The LPF used also gives a steadier performance to the proposed rectifying circuit.

The power consumption of the proposed control circuit is in the range of nanowatts between 240 and $410 \mathrm{nW}$. This is due to the simplicity of the circuit and the choice of the selected components. This allows the circuit to operate from low power conditions, which is essential in energy harvesting applications, as proven from the prototype that was integrated into an existing PMC for piezoelectric energy harvesting. The power dissipation in the diode rectifier is higher at microwatt range, especially in the FB rectifier. Therefore, it is recommended to use the VD topology for as long as possible.

\section{REFERENCES}

[1] A. Montecucco, and A. R. Knox, "Maximum power point tracking converter based on the open-circuit voltage method for thermoelectric generators," IEEE Trans. Power Electron., vol. 30, no. 2, pp. 828-839, 2015.

[2] R. Morais, S. G. Matos, M. A. Fernandes, A. L. G. Valente, S. F. S. P. Soares, P. J. S. G. Ferreira, and M. J. C. S. Reis, "Sun, wind and water flow as energy supply for small stationary data acquisition platforms," Comput. Electron. Agr., vol. 64, no. 2, pp. 120-132, Apr., 2008.

[3] Z. J. Chew, S. B. Tuddenham, and M. Zhu, "Airflow energy harvesting with high wind velocities for industrial applications," JPCS, vol. 773, no. 1, pp. 012091 (5 pp), 2016.

[4] Y. Kuang, T. Ruan, Z. J. Chew, and M. Zhu, "Energy harvesting during human walking to power a wireless sensor node," Sens. Actuat. A Phys., vol. 254, pp. 69-77, 01 Feb., 2017.

[5] M. Q. Le, J.-F. Capsal, M. Lallart, Y. Hebrard, A. Van Der Ham, N. Reffe, L. Geynet, and P.-J. Cottinet, "Review on energy harvesting for structural health monitoring in aeronautical applications," Prog. Aerosp. Sci., vol. 79, pp. 147-157, Oct., 2015.

[6] Z. J. Chew, T. Ruan, M. Zhu, M. Bafleur, and J. M. Dilhac, "Single piezoelectric transducer as strain sensor and energy harvester using time- multiplexing operation," IEEE Trans. Ind. Electron., vol. 64, no. 12, pp. 9646-9656, 2017.

[7] F. Duarte, and A. Ferreira, "Energy harvesting on railway tracks: Stateof-the-art," Proc. Inst. Civil Eng.-Transp., vol. 170, no. 3, pp. 123-130, 2017.

[8] A. C. Waterbury, and P. K. Wright, "Vibration energy harvesting to power condition monitoring sensors for industrial and manufacturing equipment," Proc. Inst. Mech. Eng. C J. Mech. Eng. Sci., vol. 227, no. 6, pp. 1187-1202, 2013.

[9] R. Bogue, "Wireless sensors: A review of technologies, products and applications," Sens. Rev., vol. 30, no. 4, pp. 285-289, 2010.

[10] M. Zhu, E. Worthington, and A. Tiwari, "Design study of piezoelectric energy-harvesting devices for generation of higher electrical power using a coupled piezoelectric-circuit finite element method," IEEE Trans. Ultrason., Ferroelectr., Freq. Control, vol. 57, no. 2, pp. 427437, 2010.

[11] Z. J. Chew, T. Ruan, and M. Zhu, "Strain energy harvesting powered wireless sensor system using adaptive and energy-aware interface for enhanced performance," IEEE Trans. Ind. Informat., vol. 13, no. 6, pp. 3006-3016, 2017.

[12] S. Fan, R. Wei, L. Zhao, X. Yang, L. Geng, and P. X. L. Feng, "An ultralow quiescent current power management system with maximum power point tracking (MPPT) for batteryless wireless sensor applications," IEEE Trans. Power Electron., vol. 33, no. 9, pp. 73267337, 2018.

[13] G. K. Ottman, H. F. Hofmann, A. C. Bhatt, and G. A. Lesieutre, "Adaptive piezoelectric energy harvesting circuit for wireless remote power supply," IEEE Trans. Power Electron., vol. 17, no. 5, pp. 669676, 2002.

[14] Y. Sun, H. H. Nguyen, C.-J. Jeong, and S.-G. Lee, "An integrated highperformance active rectifier for piezoelectric vibration energy harvesting systems," IEEE Trans. Power Electron., vol. 27, no. 2, pp. 623-627, 2012.

[15] J. Sankman, and D. Ma, "A $12-\mu \mathrm{W}$ to $1.1-\mathrm{mW}$ AIM piezoelectric energy harvester for time-varying vibrations with 450-nA $\mathrm{I}_{\mathrm{Q}}$," IEEE Trans. Power Electron., vol. 30, no. 2, pp. 632-643, 2015.

[16] S. Stanzione, C. v. Liempd, R. v. Schaijk, Y. Naito, F. Yazicioglu, and C. V. Hoof, "A high voltage self-biased integrated dc-dc buck converter with fully analog MPPT algorithm for electrostatic energy harvesters," IEEE J. Solid-State Circuits, vol. 48, no. 12, pp. 3002-3010, 2013.

[17] C. Peters, J. Handwerker, D. Maurath, and Y. Manoli, "A sub-500 mV highly efficient active rectifier for energy harvesting applications," IEEE Trans. Circuits Syst. I, Reg. Papers, vol. 58, no. 7, pp. 1542-1550, 2011.

[18] Y. S. Yuk, S. Jung, H. D. Gwon, S. Choi, S. D. Sung, T. H. Kong, S. W. Hong, J. H. Choi, M. Y. Jeong, J. P. Im, S. T. Ryu, and G. H. Cho, "An energy pile-up resonance circuit extracting maximum $422 \%$ energy from piezoelectric material in a dual-source energy-harvesting interface," in IEEE Int. Solid-State Circuits Conf. (ISSCC) Dig. Tech. Papers, 2014, pp. 402-403.

[19] A. Tabesh, and L. G. Fréchette, "A low-power stand-alone adaptive circuit for harvesting energy from a piezoelectric micropower generator," IEEE Trans. Ind. Electron., vol. 57, no. 3, pp. 840-849, 2010.

[20] Q. Tang, Q. He, M. Li, C. Dong, D. Xu, and X. Li, "Wireless alarm microsystem self-powered by vibration-threshold-triggered energy harvester," IEEE Trans. Ind. Electron., vol. 63, no. 4, pp. 2447-2456, 2016.

[21] P. Gasnier, J. Willemin, S. Boisseau, G. Despesse, C. Condemine, G. Gouvernet, and J. J. Chaillout, "An autonomous piezoelectric energy harvesting IC based on a synchronous multi-shot technique," IEEE J. Solid-State Circuits, vol. 49, no. 7, pp. 1561-1570, 2014.

[22] S. Du, Y. Jia, and A. A. Seshia, "An efficient inductorless dynamically configured interface circuit for piezoelectric vibration energy harvesting," IEEE Trans. Power Electron., vol. 32, no. 5, pp. 35953609, 2017.

[23] S. Du, Y. Jia, C. Zhao, G. A. J. Amaratunga, and A. A. Seshia, "A passive design scheme to increase the rectified power of piezoelectric energy harvesters," IEEE Trans. Ind. Electron., vol. 65, no. 9, pp. 70957105,2018

[24] Z. J. Chew, T. Ruan, and M. Zhu, "Power management circuit for wireless sensor nodes powered by energy harvesting: On the synergy of harvester and load," IEEE Trans. Power Electron., pp. 1-1, 2018.

[25] Z. J. Chew, and M. Zhu, "Adaptive maximum power point finding using direct $V_{\mathrm{OC}} / 2$ tracking method with microwatt power consumption for 
energy harvesting," IEEE Trans. Power Electron., vol. 33, no. 9, pp. 8164-8173, 2018.

[26] M. Shim, J. Kim, J. Jeong, S. Park, and C. Kim, "Self-powered $30 \mu \mathrm{W}$ to $10 \mathrm{~mW}$ piezoelectric energy harvesting system with $9.09 \mathrm{~ms} / \mathrm{V}$ maximum power point tracking time," IEEE J. Solid-State Circuits, vol. 50, no. 10, pp. 2367-2379, 2015.

[27] W. Shockley, "The theory of p-n junctions in semiconductors and p-n junction transistors," The Bell System Technical Journal, vol. 28, no. 3, pp. 435-489, 1949.

[28] Analog Devices, "LTC3388 - 20V high efficiency nanopower step-down regulator," Rep. no. LTC3388-3, Datasheet., 2010. [Online]. Available: http://www.analog.com/en/products/power-management/energyharvesting/ltc3388.html.

[29] E. E. Nigussie, "Circuit techniques for PVT variation tolerance," Variation tolerant on-chip interconnects, E. E. Nigussie, Ed. New York, NY:Springer New York, 2012, pp. 127-156.

[30] T. Hehn, F. Hagedorn, D. Maurath, D. Marinkovic, I. Kuehne, A. Frey, and Y. Manoli, "A fully autonomous integrated interface circuit for piezoelectric harvesters," IEEE J. Solid-State Circuits, vol. 47, no. 9, pp. 2185-2198, 2012.

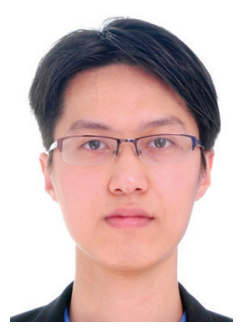

Zheng Jun Chew (M'15) received the B.Eng. degree in electronic and electrical engineering from the University of Strathclyde, Glasgow, U.K., in 2010 and the Ph.D. degree in electronic and electrical engineering from Swansea University, Swansea, U.K., in 2014.

$\mathrm{He}$ is currently a Research Fellow with the Energy Harvesting Research Group at the University of Exeter, Exeter, U.K. Prior to pursuing his Ph.D. degree, he was an Electrical Engineer at Sony EMCS(M) Sdn. Bhd., Malaysia (2010). His current research interest is in the area of power management module for energyharvesting devices using novel and low-power techniques.



Meiling Zhu (M'10) received the B.Eng. degree in mechanical manufacturing, the M.Eng. degree in Applied Mechanics and the Ph.D. degree in mechanical dynamics all from Southeast University, Nanjing, China, in 1989, 1992 and 1994.

She currently holds the Professor and the Chair in Mechanical Engineering and the Head of Energy Harvesting Research Group in the University of Exeter, Exeter, U.K. Prior to joining the University of Exeter, she worked in a number of Universities: Cranfield University (2002-2013), the University of Leeds (20012002); Stuttgart Universität (1999-2001); the Hong Kong University of Science and Technology (1998-1999); and the Institute of Vibration Engineering Research in the Nanjing University of Aeronautics and Astronautics (1994-1998). Her current research interests include the area of piezoelectric energy harvesting powered wireless sensor nodes for applications. 